\title{
Semi-Star-Alpha-Open Sets and Associated Functions
}

\author{
A. Robert \\ Department of Mathematics \\ Aditanar College of Arts and Science \\ Tiruchendur, \\ India
}

\author{
S. Pious Missier \\ P.G. Department of Mathematics \\ V.O.Chidambaram College \\ Thoothukudi \\ India
}

\begin{abstract}
The aim of this paper is to introduce various functions associated with semi* $\alpha$-open sets. Here semi* $\alpha$ continuous, semi* $\alpha$-irresolute, contra-semi ${ }^{*} \alpha$-continuous and contra-semi* $\alpha$-irresolute functions are defined. Characterizations for these functions are given. Further their fundamental properties are investigated. Many other functions associated with semi* $\alpha$-open sets and their contra versions are introduced and their properties are studied. In addition strongly semi* $\alpha$-irresolute functions, contra-strongly semi* $\alpha$-irresolute functions, semi* $\alpha$-totally continuous, totally semi* $\alpha$ continuous functions and semi* $\alpha$-homeomorphisms are introduced and their properties are investigated.
\end{abstract}

\section{General Terms: General topology}

Keywords: semi* $\alpha$-continuous, semi* $\alpha$-irresolute, semi* $\alpha$-open, semi* $\alpha$-closed, pre-semi* $\alpha$-open function, presemi* $\alpha$-closed function

\section{INTRODUCTION}

In 1963, Levine [1] introduced the concept of semicontinuity in topological spaces. Dontchev [2] introduced contra-continuous functions. Crossely and Hildebrand [3] defined pre-semi-open functions. Noiri defined and studied semi-closed functions. In 1997, Contra-open and Contraclosed functions were introduced by Baker. Dontchev and Noiri [4] introduced and studied contra-semi-continuous functions in topological spaces. Caldas [5] defined Contrapre-semi-closed functions and investigated their properties. S.Pasunkili Pandian [6] defined semi*-pre-continuous and semi*-pre-irresolute functions and their contra versions and investigated their properties. Quite recently, the authors [7, $8,9]$ introduced some new concepts, namely semi* $\alpha$-open sets, semi* $\alpha$-closed sets, the semi* $\alpha$-closure, semi* $\alpha$ derived set and semi* $\alpha$-frontier of a subset.

In this paper various functions associated with semi* $\alpha$-open sets are introduced and their properties are investigated.

\section{PRELIMINARIES}

Throughout this paper $X, Y$ and $Z$ will always denote topological spaces on which no separation axioms are assumed.

Definition 2.1[10]: A subset $A$ of a topological space $(X, \tau)$ is called (i) generalized closed (briefly g-closed) if $C l(A) \subseteq U$ whenever $A \subseteq U$ and $U$ is open .

(ii) generalized open (briefly g-open) if $X \backslash A$ is g-closed in $X$.

Definition 2.2: Let $A$ be a subset of $X$. Then (i) generalized closure[11] of $A$ is defined as the intersection of all g-closed sets containing $A$ and is denoted by $C l *(A)$. (ii) generalized interior of $A$ is defined as the union of all gopen subsets of $A$ and is denoted by $\operatorname{Int} *(A)$.

Definition 2.3: A subset $A$ of a topological space $(X, \tau)$ is (i)semi-open [1] (resp. $\alpha$-open[12], semi $\alpha$-open[13], semi-preopen[14], semi*open, semi* $\alpha$-open[7], semi*-preopen[6]) if $A \subseteq C l(\operatorname{Int}(A))$ (resp. $A \subseteq \operatorname{Int}(C l(\operatorname{Int}(A)), A \subseteq C l(\operatorname{Int}(C l(\operatorname{Int}(A))), A \subseteq C l(\operatorname{Int}(C l(A)))$, $A \subseteq C l^{*}(\operatorname{Int}(A)), A \subseteq C l^{*}\left(\alpha \operatorname{Int}(A), A \subseteq C l^{*}(\operatorname{pInt}(A)),\right)$ (ii)semi-closed (resp. $\alpha$-closed[12], semi $\alpha$-closed[15], semi-preclosed[14], semi*-closed, semi* $\alpha$-closed[8], semi*-preclosed[6]) if $\operatorname{Int}(C l(A)) \subseteq A$ (resp. $\operatorname{Cl}(\operatorname{Int}(C l(A))) \subseteq A, \operatorname{Int}(C l(\operatorname{Int}(C l(A)) \subseteq A$, Int $*(C l(A)) \subseteq A, \quad \operatorname{Int} *(\alpha C l(A)) \subseteq A, \operatorname{Int} *(p C l(A)) \subseteq A)$. (iii) semi* $\alpha$-regular $[8]$ if it is both semi* $\alpha$-open and semi* $\alpha$-closed.

Definition 2.4: Let $A$ be a subset of $X$. Then (i)The semi* $\alpha$-interior [7] of $A$ is defined as the union of all semi* $\alpha$-open subsets of $A$ and is denoted by $s^{*} \alpha \operatorname{Int}(A)$.

(ii) The semi* $\alpha$-closure [8] of $A$ is defined as the intersection of all semi* $\alpha$-closed sets containing $A$ and is denoted by $s^{*} \alpha C l(A)$.

Definition 2.5: A function $f: X \rightarrow Y$ is said to be semicontinuous [1] (resp. contra-semi-continuous [4], semi*continuous, contra-semi*-continuous, semi $\alpha$-continuous [15] ) if $f^{1}(V)$ is semi-open(resp. semi-closed, semi*-open, semi*closed, semi $\alpha$-open) in $X$ for every open set $V$ in $Y$.

Definition 2.6: A function $f: X \rightarrow Y$ is said to be $\alpha$-continuous (resp. semi-pre-continuous [14], semi*-pre-continuous [6]) if $f^{1}(V)$ is $\alpha$-open(resp. semi-preopen, semi*-preopen) in $X$ for every open set $V$ in $Y$.

Definition 2.7: A topological space $X$ is said to be

(i) $\mathrm{T}_{1 / 2}$ if every g-closed set in $X$ is closed.[10]

(ii) locally indiscrete if every open set is closed.

Theorem 2.8: [7] (i) Every $\alpha$-open set is semi* $\alpha$-open.

(ii)Every open set is semi* $\alpha$-open.

(iii)Every semi*-open set is semi* $\alpha$-open.

(iv)Every semi* $\alpha$-open set is semi $\alpha$-open.

(v)Every semi* $\alpha$-open set is semi*-preopen.

(vi)Every semi* $\alpha$-open set is semi-preopen.

(vii)Every semi* $\alpha$-open set is semi-open.

Remark 2.9:[8] Similar results for semi* $\alpha$-closed sets are also true.

Theorem 2.10: [7] (i) Arbitrary union of semi* $\alpha$-open sets is also semi* $\alpha$-open. 
(ii) If $A$ is semi* $\alpha$-open in $X$ and $B$ is open in $X$, then $A \cap B$ is semi* $^{*} \alpha$-open in $X$.

(iii)A subset $A$ of a space $\mathrm{X}$ is semi* $\alpha$-open if and only if $s^{*} \alpha \operatorname{Int}(A)=A$.

Theorem 2.11: [7] For a subset $A$ of a space $\mathrm{X}$ the following are equivalent:

(i) $A$ is semi* $\alpha$-open in $\mathrm{X}$

(ii) $A \subseteq C l^{*}(\alpha \operatorname{Int}(A))$.

(iii) $C l^{*}(\alpha \operatorname{Int}(A))=C l^{*}(A)$.

Theorem 2.12: [8]For a subset $A$ of a space $\mathrm{X}$ the following are equivalent:

(i) $A$ is semi* $\alpha$-closed in $\mathrm{X}$.

(ii) $\operatorname{Int} *(\alpha C l(A)) \subseteq A$

(iii) $\operatorname{Int} *(\alpha C l(A))=\operatorname{Int} *(A)$.

Theorem 2.13: [8] (i) A subset $A$ of a space $X$ is semi* $\alpha$ closed if and only if $s^{*} \alpha C l(A)=A$.

(ii)Let $A \subseteq X$ and let $x \in X$. Then $x \in s^{*} \alpha C l(A)$ if and only if every semi* $\alpha$-open set in $X$ containing $X$ intersects $A$.

Definition 2.14: [9] If $A$ is a subset of $X$, the semi* $\alpha$-Frontier of $A$ is defined by

$\mathrm{s}^{*} \alpha \operatorname{Fr}(A)=s^{*} \alpha \operatorname{Cl}(A) \backslash s * \alpha \operatorname{Int}(A)$.

Theorem 2.15:[9] If $A$ is a subset of $X$, then $\mathrm{s}^{*} \alpha \operatorname{Fr}(A)=s^{*} \alpha C l(A) \cap s^{*} \alpha C l(X \backslash A)$

Definition 2.16:[15] A function $f: X \rightarrow Y$ is said to be (i) semi $\alpha^{*}$-continuous (resp. semi $\alpha^{* *}$-continuous ) if $f^{1}(V)$ is semi $\alpha$-open(resp. open) set in $X$ for every semi $\alpha$-open set $V$ in $Y$. (ii) totally semi-continuous [16] if $f^{1}(V)$ is semi regular in $X$ for every open set $V$ in $Y$.

(iii) semi-totally continuous [17] if $f^{1}(V)$ is clopen in $X$ for every semi-open set $V$ in $Y$.

\section{SEMI* $\alpha$-CONTINUOUS FUNCTIONS}

In this section we define the semi* $\alpha$-continuous and contra-semi* $\alpha$-continuous functions and investigate their fundamental properties

Definition 3.1: A function $f: X \rightarrow Y$ is said to be semi* $\alpha$ continuous at $x \in X$ if for each open set $V$ of $Y$ containing $f(x)$, there is a semi* $\alpha$-open set $U$ in $X$ such that $x \in U$ and $f(U) \subseteq V$.

Definition 3.2: A function $f: X \rightarrow Y$ is said to be semi* $\alpha$ continuous if $f^{-1}(V)$ is semi* $\alpha$-open in $X$ for every open set $V$ in $Y$.

Theorem 3.3: Let $f: X \rightarrow Y$ be a function. Then the following statements are equivalent:

(i) $f$ is semi* $\alpha$-continuous.

(ii) $f$ is semi* $\alpha$-continuous at each point $x \in X$.

(iii) $f^{-1}(F)$ is semi* $\alpha$-closed in $X$ for every closed set $F$ in $Y$.

(iv) $f\left(s^{*} \alpha C l(A)\right) \subseteq C l(f(A))$ for every subset $A$ of $X$.

(v) $s^{*} \alpha C l\left(f^{-1}(B)\right) \subseteq f^{-1}(C l(B))$ for every subset $B$ of $Y$.

(vi) $f^{-1}(\operatorname{Int}(B)) \subseteq S^{*} \alpha \operatorname{Int}\left(f^{-1}(B)\right)$ for every subset $B$ of $Y$.

(vii) $\operatorname{Int} *\left(\alpha C l\left(f^{-1}(F)\right)\right)=\operatorname{In} t^{*}\left(f^{-1}(F)\right)$ for every closed set $F$ in $Y$. (viii) $C l^{*}\left(\alpha \operatorname{Int}\left(f^{-1}(V)\right)\right)=C l^{*}\left(f^{-1}(V)\right)$ for every open set $V$ in $Y$.

Proof: (i) $\Rightarrow$ (ii): Let $f: X \rightarrow Y$ be semi* $\alpha$-continuous. Let $x \in X$ and $V$ be an open set in $Y$ containing $f(x)$. Then $x \in f^{-1}(V)$. Since $f$ is semi* $\alpha$-continuous, $U=f^{-1}(V)$ is a semi* $\alpha$ open set in $X$ containing $x$ such that $f(U) \subseteq V$. (ii) $\Rightarrow$ (i): Let $f: X \rightarrow Y$ be semi* $\alpha$-continuous at each point of $X$. Let $\mathrm{V}$ be an open set in $\mathrm{Y}$. Let $x \in f^{-1}(V)$. Then $\mathrm{V}$ is an open set in Y containing $f(x)$. By (ii), there is a semi* $\alpha$-open set $U_{x}$ in $X$ containing $x$ such that $f(x) \in f\left(U_{x}\right) \subseteq V$. Therefore $U_{x} \subseteq f$ ${ }^{1}(V)$. Hence $f^{-1}(V)=\cup\left\{U_{x}: x \in f^{-1}(V)\right\}$. By Theorem 2.10(i), $f$ ${ }^{-1}(V)$ is semi* $\alpha$-open in $X$.

(i) $\Rightarrow$ (iii): Let $F$ be a closed set in $Y$. Then $V=Y N F$ is open in $Y$. Then $f^{-1}(V)$ is semi* $\alpha$-open in $\mathrm{X}$. Therefore

$f^{-1}(F)=f^{-1}(Y V)=X \backslash f^{-1}(V)$ is semi* $\alpha$-closed.

(iii) $\Rightarrow(\mathbf{i})$ : $\quad$ Let $\mathrm{V}$ be an open set in $\mathrm{Y}$. Then $\mathrm{F}=\mathrm{Y} \backslash \mathrm{V}$ is semi* $\alpha$ closed. By (iii), $f^{-1}(F)$ is semi* $\alpha$-closed. Hence $f^{-1}(V)=f^{-1}(Y F)=X \backslash f^{-1}(F)$ is semi* $\alpha$-open in $X$.

(iii) $\Rightarrow$ (iv): Let $A \subseteq X$. Let $F$ be a closed set containing $f(A)$. Then by (iii), $f^{-1}(F)$ is a semi* $\alpha$-closed set containing $A$. This implies that $s^{*} \alpha C l(A) \subseteq f^{1}(F)$ and hence $f\left(s^{*} \alpha C l(A)\right) \subseteq F$.

(iv) $\Rightarrow(\mathbf{v})$ : Let $B \subseteq Y$ and let $A=f^{-1}(B)$. By assumption, $f\left(s^{*} \alpha C l(A)\right) \subseteq C l(f(A)) \subseteq C l(B)$. This implies that

$s^{*} \alpha C l(A) \subseteq f^{-1}(C l(B))$.

(v) $\Rightarrow$ (iii): Let $\mathrm{F}$ be closed in Y. Then $C l(B)=B$. Therefore (v) implies $s^{*} \alpha C l\left(f^{-1}(B)\right) \subseteq f^{1}(B)$. Hence $s^{*} \alpha C l\left(f^{-1}(B)\right)=$ $f^{-1}(B)$. By Theorem 2.13(i), $f^{-1}(B)$ is semi* $\alpha$-closed.

$(\mathbf{v}) \Leftrightarrow(\mathbf{v i})$ : The equivalence of (v) and (vi) can be proved by taking the complements.

(vii) $\Leftrightarrow($ iii): Follows from Theorem 2.12.

(viii) $\Leftrightarrow(i):$ Follows from Theorem 2.11.

Theorem 3.4: (i) Every $\alpha$-continuous function is $\operatorname{semi}^{*} \alpha$ continuous.

(ii) Every continuous function is semi* $\alpha$-continuous

(iii) Every constant function is semi* $\alpha$-continuous.

(iv) Every semi* $\alpha$-continuous function is semi $\alpha$-continuous.

(v) Every semi* $\alpha$-continuous function is semi* precontinuous.

(vi) Every semi* $\alpha$-continuous function is semi-precontinuous

(vii) Every semi*-continuous function is semi* $\alpha$-continuous.

(viii) Every semi*-continuous function is semi-continuous.

Proof: Follows from Theorem 2.8 and the fact that every constant function is continuous.

Remark 3.5: In general the converse of each of the statements in Theorem 3.4 is not true.

Theorem 3.6: If the topology of the space $Y$ is given by a basis $\mathrm{B}$, then a function $f: X \rightarrow Y$ is semi* $\alpha$-continuous if and only if the inverse image of every basic open set in $Y$ under $f$ is semi* $\alpha$-open in $X$.

Proof: Suppose $f: X \rightarrow Y$ is semi* $\alpha$-continuous. Then inverse image of every open set in $Y$ is semi* $\alpha$-open in $X$. In particular, inverse image of every basic open set in $Y$ is semi* $\alpha$-open in $X$. Conversely, let $V$ be an open set in $Y$. Then $V=\cup B_{\mathrm{i}}$ where $B_{\mathrm{i}} \in \mathrm{B}$. Now $f^{-1}(V)=f^{-1}\left(\cup B_{\mathrm{i}}\right)=\cup f^{-1}\left(B_{\mathrm{i}}\right)$. By hypothesis, $f^{-1}\left(B_{\mathrm{i}}\right)$ is semi* $\alpha$-open for each i. By Theorem 2.10(i), $f$ ${ }^{1}(V)=\cup f^{-1}\left(B_{\mathrm{i}}\right)$ is semi* $\alpha$-open. Hence $f$ is semi* $\alpha$-continuous.

Theorem 3.7: A function $f: X \rightarrow Y$ is not semi* $\alpha$-continuous at point $x \in X$ if and only if $x$ belongs to the semi* $\alpha$-frontier of the inverse image of some open set in $Y$ containing $f(x)$.

Proof: Suppose $f$ is not semi* $\alpha$-continuous at $x$. Then by Definition 3.1, there is an open set $V$ in $Y$ containing $f(x)$ such that $f(U)$ is not a subset of $V$ for every semi* $\alpha$-open set $U$ in $X$ containing $x$. Hence $U \cap\left(X \backslash f^{1}(V)\right) \neq \phi$ for every semi* $\alpha$-open set $U$ containing $x$. By Theorem 2.13(ii), we get $x \in s^{*} \alpha C l\left(X \backslash f^{-}\right.$ $\left.{ }^{1}(V)\right)$. Also $x \in f^{-1}(V) \subseteq s^{*} \alpha C l\left(f^{-1}(V)\right)$. Hence $x \in s^{*} \alpha \mathrm{Cl}(f$ 
$\left.{ }^{1}(V)\right) \cap s^{*} \alpha C l\left(X \backslash f^{1}(V)\right)$. By Theorem 2.15, $x \in \mathrm{s}^{*} \alpha F \mathrm{r}\left(f^{-1}(V)\right)$. On the other hand, let $f$ be semi* ${ }^{*} \alpha$-continuous at $x \in X$. Let $V$ be any open set in $Y$ containing $f(x)$. Then there exists a semi* $\alpha$ open set $U$ in $X$ containing $x$ such that $f(U) \subseteq V$. That is, $U$ is a semi* $\alpha$-open set in $X$ containing $x$ such that $U \subseteq f^{-1}(V)$. Hence $x \in S^{*} \alpha \operatorname{Int}\left(f^{-1}(V)\right)$. Therefore by Definition 2.14, $x \notin \mathrm{s}^{*} \alpha F \mathrm{r}(f$ $\left.{ }^{1}(V)\right)$.

Theorem 3.8: Let $f: X \rightarrow \Pi X_{\alpha}$ be semi* $\alpha$-continuous where $\Pi X_{\alpha}$ is given the product topology and $f(x)=\left(f_{\alpha}(x)\right)$. Then each coordinate function $f_{\alpha}: X \rightarrow X_{\alpha}$ is semi* $\alpha$-continuous.

Proof: Let $V$ be an open set in $X_{\alpha}$. Then $f_{\alpha}^{-1}(V)=\left(\pi_{\alpha} \circ f\right)^{-1}(V)=f^{-1}\left(\pi_{\alpha}^{-1}(V)\right)$, where

$\pi_{\alpha}: \Pi X_{\alpha} \rightarrow X_{\alpha}$ is the projection map. Since is $\pi_{\alpha}$ continuous, $\pi_{\alpha}{ }^{-}$ ${ }^{1}(V)$ is open in $\Pi X_{\alpha}$. By the semi* $\alpha$-continuity of $f, \quad f_{\alpha}$ ${ }^{1}(V)=f^{1}\left(\pi_{\alpha}^{-1}(V)\right)$ is semi* $\alpha$-open in $X$. Therefore $f_{\alpha}$ is semi* $\alpha$ continuous.

Theorem 3.9: Let $f: X \rightarrow \Pi X_{\alpha}$ be defined by $f(x)=\left(f_{\alpha}(x)\right)$ and $\Pi X_{\alpha}$ be given the product topology. Suppose $\mathrm{S}^{*} \alpha \mathrm{O}(X)$ is closed under finite intersection. Then $f$ is semi* $\alpha$-continuous if each coordinate function $f_{\alpha}: X \longrightarrow X_{\alpha}$ is semi* $\alpha$-continuous,

Proof: $\quad$ Let $V$ be a basic open set in $\Pi X_{\alpha}$. Then

$V=\cap \pi_{\alpha}{ }^{-1}\left(V_{\alpha}\right)$ where each $V_{\alpha}$ is open in $X_{\alpha}$, the intersection being taken over finitely many $\alpha$ 's. Now $f^{-1}(V)=$ $f^{-1}\left(\cap \pi_{\alpha}{ }^{-1}\left(V_{\alpha}\right)\right)=\bigcap\left(f^{-1}\left(\pi_{\alpha}^{-1}\left(V_{\alpha}\right)\right)\right)=\bigcap\left(\pi_{\alpha} \circ f\right)^{-1}(V)=\cap f_{\alpha}^{-1}(V)$ is semi* $^{*} \alpha$-open, by hypothesis. Hence by Theorem 3.6, $f$ is semi* $\alpha$-continuous.

Theorem 3.10: Let $f: X \rightarrow Y$ be continuous and $g: X \rightarrow Z$ be semi* $\alpha$-continuous. Let $h: X \rightarrow Y \times Z$ be defined by $h(x)=(f(x)$, $g(x))$ and $Y \times Z$ be given the product topology. Then $h$ is semi* $\alpha$-continuous.

Proof: By virtue of Theorem 3.6, it is sufficient to show that inverse image under $h$ of every basic open set in $Y \times Z$ is semi* $\alpha$-open in $X$. Let $U \times V$ be a basic open set in $Y \times Z$. Then $h^{-1}(U \times V)=f^{-1}(U) \cap g^{-1}(V)$. By continuity of $f, f^{-1}(U)$ is open in $X$ and by semi* $\alpha$-continuity of $g, g^{-1}(V)$ is semi* $\alpha$-open in $X$. By invoking Theorem 2.10(ii), we get $h^{-1}(U \times V)=f^{-1}(U) \cap g$ ${ }^{1}(V)$ is semi* $\alpha$-open

Remark 3.11: The above theorem is true even if $f$ is semi* $\alpha$ continuous and $g$ is continuous.

Theorem 3.12: Let $f: X \rightarrow Y$ be semi* $\alpha$-continuous and $g: Y \rightarrow Z$ be continuous.

Then $g \circ f: X \rightarrow Z$ is semi* $\alpha$-continuous.

Proof: Let $V$ be an open set in $Z$. Since $g$ is continuous, $g$

${ }^{1}(V)$ is open in $Y$. By semi* $\alpha$-continuity of $f,(g \circ f)^{-1}(V)=f^{1}(g$

$\left.{ }^{1}(V)\right)$ is semi* $\alpha$-open in $X$. Hence $g \circ f$ is semi* $\alpha$-continuous.

Remark 3.13: Composition of two semi* $\alpha$-continuous functions need not be semi* $\alpha$-continuous.

Definition 3.14: A function $f: X \rightarrow Y$ is called contra-semi* $\alpha$ continuous if $f^{1}(V)$ is semi* $\alpha$ - closed in $X$ for every open set $V$ in $Y$.

Theorem 3.15: For a function $f: X \rightarrow Y$, the following are equivalent:

(i) $f$ is contra-semi* ${ }^{*} \alpha$-continuous.

(ii) For each $x \in X$ and each closed set $F$ in $Y$ containing $f(x)$, there exists a semi* ${ }^{*} \alpha$-open set $U$ in $X$ containing $x$ such that $f(U) \subseteq F$.
(iii)The inverse image of each closed set in $Y$ is semi* $\alpha$-open in $X$. (iv) $C l^{*}\left(\alpha \operatorname{Int}\left(f^{-1}(F)\right)\right)=C l^{*}\left(f^{-1}(F)\right)$ for every closed set $F$ in $Y$. (v) $\operatorname{Int} *\left(\alpha C l\left(f^{1}(V)\right)\right)=\operatorname{In} t^{*}\left(f^{1}(V)\right)$ for every open set $V$ in $Y$.

Proof: (i) $\Rightarrow$ (ii): Let $f: X \rightarrow Y$ be contra-semi* $\alpha$-continuous. Let $\mathrm{x} \in X$ and $F$ be a closed set in $Y$ containing $f(x)$. Then $V=Y \backslash F$ is an open set in $Y$ not containing $f(x)$. Since $f$ is contrasemi* $\alpha$-continuous, $f^{-1}(V)$ is a semi* $\alpha$-closed set in $X$ not containing $x$. That is, $f^{-1}(V)=X \backslash f^{-1}(F)$ is a semi* $\alpha$-closed set in $X$ not containing $x$. Therefore

$U=f^{1}(F)$ is a semi* $\alpha$-open set in $X$ containing $x$ such that $f(U) \subseteq F$.

(ii) $\Rightarrow$ (iii): Let $F$ be a closed set in $Y$. Let $x \in f^{-1}(F)$, then $f(x) \in F$. By (ii), there is a semi* $\alpha$-open set $U_{x}$ in $X$ containing $x$ such that $f(x) \in f\left(U_{x}\right) \subseteq F$. That is, $x \in U_{x} \subseteq$

$f^{-1}(F)$. Therefore $f^{-1}(F)=\cup\left\{U_{x}: x \in f^{-1}(F)\right\}$. By Theorem 2.10(i), $f^{-1}(F)$ is semi* $\alpha$-open in $X$. (iii) $\Rightarrow$ (iv): Let $F$ be a closed set in $Y$. By (iii), $f^{-1}(F)$ is a semi* $\alpha$-open set in $X$. By Theorem 2.11, $C l^{*}\left(\alpha \operatorname{Int}\left(f^{-1}(F)\right)\right)=C l^{*}\left(f^{-1}(F)\right)$.

(iv) $\Rightarrow(\mathbf{v})$ : If $V$ is any open set in $Y$, then $Y V$ is closed in $Y$. By (iv), we have $C l^{*}\left(\alpha \operatorname{Int}\left(f^{-1}(Y V)\right)\right)=C l^{*}\left(f^{-1}(Y \backslash V)\right)$. Taking the complements, we get $\operatorname{In} t^{*}\left(\alpha C l\left(f^{-1}(V)\right)\right)=\operatorname{In} t^{*}\left(f^{-1}(V)\right)$.

(v) $\Rightarrow(\mathbf{i})$ : Let $V$ be any open set in $Y$. Then by assumption, $\operatorname{Int} *\left(\alpha C l\left(f^{-1}(V)\right)\right)=\operatorname{Int} *\left(f^{-1}(V)\right) . \quad$ By Theorem 2.12, $f^{-1}(V)$ is semi* $\alpha$-closed .

Theorem 3.16: Every contra- $\alpha$-continuous function is contrasemi* $\alpha$-continuous.

Proof: Follows from Remark 2.9

Remark 3.17: It can be seen that the converse of the above theorem is not true.

Theorem 3.18: Every contra-semi* $\alpha$-continuous function is contra-semi $\alpha$-continuous.

Proof: Let $f: X \rightarrow Y$ be contra-semi* $\alpha$-continuous. Let $V$ be an open set in $Y$. Since $f$ is contra-semi* $\alpha$-continuous, $f^{-1}(V)$ is semi* $\alpha$-closed in $X$. By Remark $2.9, f^{-1}(V)$ is semi$\alpha$-closed in $X$. Hence $f$ is contra-semi- $\alpha$-continuous.

Remark 3.19: It can be easily seen that the converse of the above theorem is not true.

Composition of two contra-semi* $\alpha$-continuous functions need not be contra-semi* $\alpha$-continuous.

\section{SEMI* $\alpha$-IRRESOLUTE FUNCTIONS}

In this section we define the semi* $\alpha$-irresolute and contra-semi* $\alpha$-irresolute functions and investigate their fundamental properties.

Definition 4.1: A function $f: X \rightarrow Y$ is said to be semi* $\alpha$ irresolute at $x \in X$ if for each semi* $\alpha$-open set $V$ of $Y$ containing $f(x)$, there is a semi* $\alpha$-open set $U$ of $X$ such that $x \in U$ and $f(U) \subseteq V$.

Definition 4.2: A function $f: X \rightarrow Y$ is said to be semi* $\alpha$ irresolute if $f^{-1}(V)$ is semi* $\alpha$-open in $X$ for every semi* $\alpha$-open set $V$ in $Y$.

Definition 4.3: A function $f: X \rightarrow Y$ is said to be contrasemi* $\alpha$-irresolute if $f^{-1}(V)$ is semi* $\alpha$-closed in $X$ for every semi* ${ }^{*} \alpha$-open set $V$ in $Y$. 
Definition 4.4: A function $f: X \rightarrow Y$ is said to be strongly semi* $\alpha$-irresolute if $f^{-1}(V)$ is open in $X$ for every semi* $\alpha$-open set $V$ in $Y$.

Definition 4.5: A function $f: X \rightarrow Y$ is said to be contrastrongly semi* $\alpha$-irresolute if $f^{-1}(V)$ is closed in $X$ for every semi* $\alpha$-open set $V$ in $Y$.

Theorem 4.6: Every semi* $\alpha$-irresolute function is $\operatorname{semi}^{*} \alpha-$ continuous.

Proof: $\quad$ Let $f: X \rightarrow Y$ be semi* $\alpha$-irresolute. Let $V$ be open in $Y$. Then by Theorem 2.8(ii), $V$ is semi* $\alpha$-open. Since $f$ is semi* $\alpha$-irresolute, $f^{1}(V)$ is semi* $\alpha$-open in $X$. Thus $f$ is semi* $\alpha$-continuous.

Theorem 4.7: Every constant function is semi* $\alpha$-irresolute. Proof: Let $f: X \rightarrow Y$ be a constant function defined by $f(x)=y_{0}$ for all $x$ in $X$, where $y_{0}$ is a fixed point in $Y$. Let $V$ be a semi* $\alpha$-open set in $Y$. Then $f^{-1}(V)=X$ or $\phi$ according as $y_{0} \in V$ or $y_{0} \notin V$. Thus $f^{-1}(V)$ is semi* $\alpha$-open in $X$. Hence $f$ is semi* $\alpha$-irresolute.

Theorem 4.8: Let $f: X \rightarrow Y$ be a function. Then the following are equivalent:

(i) $f$ is semi* $\alpha$-irresolute.

(ii) $f$ is semi* $\alpha$-irresolute at each point of $X$.

(iii) $f^{-1}(F)$ is semi* $\alpha$-closed in $X$ for every semi* $\alpha$-closed set $F$ in $Y$.

(iv) $f\left(s^{*} \alpha C l(A)\right) \subseteq s^{*} \alpha C l(f(A))$ for every subset $A$ of $X$.

(v) $s^{*} \alpha C l\left(f^{1}(B)\right) \subseteq f^{1}\left(s^{*} \alpha C l(B)\right)$ for every subset $B$ of $Y$.

(vi) $f^{-1}\left(s^{*} \alpha \operatorname{Int}(B)\right) \subseteq s^{*} \alpha \operatorname{Int}\left(f^{-1}(B)\right)$ for every subset $B$ of $Y$.

(vii) $\operatorname{Int} *\left(\alpha C l\left(f^{-1}(F)\right)\right)=\operatorname{Int} *\left(f^{-1}(F)\right)$ for every semi* $\alpha$-closed set $F$ in $Y$.

(viii) $C l^{*}\left(\alpha \operatorname{Int}\left(f^{-1}(V)\right)\right)=C l^{*}\left(f^{-1}(V)\right)$ for every semi* $\alpha$-open set $V$ in $Y$.

Proof: (i) $\Rightarrow$ (ii): Let $f: X \rightarrow Y$ be semi* $\alpha$-irresolute. Let $x \in X$ and $V$ be a semi* $\alpha$-open set in $Y$ containing $f(x)$. Then $x \in f^{-1}(V)$. Since $f$ is semi* $\alpha$-irresolute, $U=f^{-1}(V)$ is a semi* $\alpha$ open set in $X$ containing $x$ such that $f(U) \subseteq V$.

(ii) $\Rightarrow$ (i): Let $f: X \rightarrow Y$ be semi* $\alpha$-irresolute at each point of $X$. Let $\mathrm{V}$ be a semi* $\alpha$-open set in $\mathrm{Y}$. Let $x \in f^{-1}(V)$. Then $\mathrm{V}$ is a semi* $\alpha$-open set in $\mathrm{Y}$ containing $f(x)$. By (ii), there is a semi* $\alpha$-open set $U_{x}$ in $X$ containing $x$ such that $f(x) \in f\left(U_{x}\right) \subseteq V$. Therefore $U_{x} \subseteq f^{-1}(V)$. Hence $f^{-1}(V)=\cup\left\{U_{x}: x \in f^{-1}(V)\right\}$. By Theorem 2.10(i), $f^{-1}(V)$ is semi* $\alpha$-open in $X$.

(i) $\Rightarrow$ (iii): Let $F$ be a semi* $\alpha$-closed set in $Y$. Then $V=Y \backslash F$ is semi* ${ }^{*} \alpha$-open in $Y$. Then $f^{-1}(V)$ is semi* $\alpha$-open in $\mathrm{X}$. Therefore $f^{-1}(F)=f^{-1}(Y V)=X \backslash f^{-1}(V)$ is semi* $\alpha$-closed.

(iii) $\Rightarrow$ (i): $\quad$ Let $\mathrm{V}$ be a semi* $\alpha$-open set in $\mathrm{Y}$. Then $\mathrm{F}=\mathrm{Y} \backslash \mathrm{V}$ is semi* $\alpha$-closed. By (iii), $f^{-1}(F)$ is semi* $\alpha$-closed. Hence

$f^{-1}(V)=f^{-1}(Y F)=X \backslash f^{-1}(F)$ is semi* $\alpha$-open in $X$.

(iii) $\Rightarrow$ (iv): Let $A \subseteq X$. Let $F$ be a semi* $\alpha$-closed set containing $f(A)$. Then by (iii), $f^{-1}(F)$ is a semi* $\alpha$-closed set containing $A$. This implies that $s^{*} \alpha C l(A) \subseteq f^{1}(F)$ and hence $f\left(s^{*} \alpha C l(A)\right) \subseteq F$. Therefore $f\left(s^{*} \alpha C l(A)\right) \subseteq C l(f(A))$.

(iv) $\Rightarrow(\mathbf{v})$ : Let $B \subseteq Y$ and let $A=f^{-1}(B)$. By assumption, $f\left(s^{*} \alpha C l(A)\right) \subseteq \mathrm{s}^{*} \alpha C l(f(A)) \subseteq \mathrm{s} * \alpha C l(B)$. This implies that $s^{*} \alpha C l(A) \subseteq f^{-1}\left(\mathrm{~s}^{*} \alpha C l(B)\right)$. Hence $s^{*} \alpha C l\left(f^{-1}(B)\right) \subseteq f^{-1}\left(\mathrm{~s}^{*} \alpha C l(B)\right)$.

(v) $\Rightarrow$ (iii): Let $\mathrm{F}$ be semi* $\alpha$-closed in Y. Then $\mathrm{s}^{*} \alpha C l(F)=F$. Therefore (v) implies $s^{*} \alpha C l\left(f^{-1}(F)\right) \subseteq f^{-1}(F)$. Hence $s^{*} \alpha C l\left(f^{-1}(F)\right)=f^{-1}(F)$. By Theorem 2.13(i), $f^{-1}(F)$ is semi* $\alpha$ closed. $(\mathbf{v}) \Leftrightarrow(\mathbf{v i})$ : The equivalence of (v) and (vi) can be proved by taking the complements.

(vii) $\Leftrightarrow($ iii): Follows from Theorem 2.12.

(viii) $\Leftrightarrow(\mathbf{i})$ :Follows from Theorem 2.11.

Theorem 4.9: Let $f: X \rightarrow Y$ be a function. Then $f$ is not semi* $\alpha$-irresolute at a point $x$ in $X$ if and only if $x$ belongs to the semi* $\alpha$-frontier of the inverse image of some semi* $\alpha$-open set in $Y$ containing $f(x)$.

Proof: Suppose $f$ is not semi* $\alpha$-irresolute at $x$. Then by Definition 4.1, there is a semi* $\alpha$-open set $V$ in $Y$ containing $f(x)$ such that $f(U)$ is not a subset of $V$ for every semi* $\alpha$-open set $U$ in $X$ containing $x$. Hence $U \cap\left(X \backslash f^{-1}(V)\right) \neq \phi$ for every semi* $\alpha$ open set $U$ containing $x$. Thus

$x \in s^{*} \alpha C l\left(X \backslash f^{-1}(V)\right)$. Since $x \in f^{1}(V) \subseteq s^{*} \alpha C l\left(f^{-1}(V)\right)$, we have $x \in s^{*} \alpha C l\left(f^{-1}(V)\right) \cap s^{*} \alpha C l\left(X \backslash f^{-1}(V)\right)$. Hence by Theorem 2.15, $x \in \mathrm{s}^{*} \alpha F \mathrm{r}\left(f^{-1}(V)\right)$. On the other hand, let $f$ be semi* $\alpha$-irresolute at $x$. Let $V$ be a semi* $\alpha$-open set in $Y$ containing $f(x)$. Then there is a semi*a-open set $U$ in $X$ containing $x$ such that $f(x) \in f(U) \subseteq V$. Therefore $U \subseteq f^{-1}(V)$. Hence

$x \in S^{*} \alpha \operatorname{Int}\left(f^{-1}(V)\right)$. Therefore by Definition 2.14,

$x \notin \mathrm{S}^{*} \alpha \mathrm{Fr}\left(f^{-1}(V)\right)$ for every open set $V$ containing $f(x)$.

Theorem 4.10: Every contra-semi* $\alpha$-irresolute function is contra-semi* $\alpha$-continuous.

Proof: Let $f: X \rightarrow Y$ be a contra-semi* $\alpha$-irresolute function. Let $V$ be an open set in $Y$. Then by Theorem 2.8(ii), $V$ is semi* $^{*} \alpha$-open in $Y$. Since $f$ is contra-semi* $\alpha$-irresolute,

$f^{-1}(V)$ is semi* $\alpha$-closed in $X$. Hence $f$ is contra-semi* $\alpha$ continuous.

Theorem 4.11: For a function $f: X \rightarrow Y$, the following are equivalent:

(i) $\quad f$ is contra-semi* $\alpha$-irresolute.

(ii) The inverse image of each semi* $\alpha$-closed set in $Y$ is semi* $\alpha$-open in $X$.

(iii) For each $x \in X$ and each semi* $\alpha$-closed set $F$ in $Y$ with $f(x) \in F$, there exists a semi* $\alpha$-open set $U$ in $X$ such that $x \in U$ and $f(U) \subseteq F$.

(iv) $C l^{*}\left(\alpha \operatorname{Int}\left(f^{-1}(F)\right)\right)=C l^{*}\left(f^{-1}(F)\right)$ for every semi* $\alpha$-closed set $F$ in $Y$.

(v) $\operatorname{In} t^{*}\left(\alpha C l\left(f^{-1}(V)\right)\right)=\operatorname{Int} t^{*}\left(f^{-1}(V)\right)$ for every semi* $\alpha$-open set $V$ in $Y$.

Proof:(i) $\Rightarrow($ ii): Let $F$ be a semi* $\alpha$-closed set in $Y$. Then $Y F$ is semi* $\alpha$-open in $Y$. Since $f$ is contra-semi* $\alpha$-irresolute, $f$ ${ }^{1}(Y F)=$

$X \backslash f^{-1}(F)$ is semi* $\alpha$-closed in $X$.

(ii) $\Rightarrow$ (iii): Let $F$ be a semi* $\alpha$-closed set in $Y$ containing $f(x)$. Then $U=f^{-1}(F)$ is a semi* $\alpha$-open set containing $x$ such that $f(U) \subseteq F$.

(iii) $\Rightarrow$ (iv): Let $F$ be a semi* ${ }^{*} \alpha$-closed set in $Y$ and $x \in f^{-1}(F)$, then $f(x) \in F$. By assumption, there is a semi* $\alpha$-open set $U_{x}$ in $X$ containing $x$ such that $f(x) \in f\left(U_{x}\right) \subseteq F$ which implies that $x \in U_{x} \subseteq f^{-1}(F)$. This follows that $f^{-1}(F)=$

$\cup\left\{U_{x}: x \in f^{-1}(F)\right\}$. By Theorem 2.10(i), $f^{-1}(F)$ is semi* $\alpha$ open in $X$. By Theorem 2.11, $C l^{*}\left(\alpha \operatorname{Int}\left(f^{-1}(F)\right)\right)=C l^{*}\left(f^{-1}(F)\right)$.

(iv) $\Rightarrow(\mathbf{v})$ : Let $V$ be a semi* $\alpha$-open set in $Y$. Then $Y V$ is semi* $\alpha$-closed in $Y$. By assumption, $C l^{*}\left(\alpha \operatorname{Int}\left(f^{-1}(\Lambda V)\right)\right)=$ $C l^{*}\left(f^{-1}(Y \backslash)\right)$. Taking the complements we get, $\operatorname{Int} t^{*}\left(\alpha C l\left(f^{-1}(V)\right)\right)=\operatorname{In} t^{*}\left(f^{-1}(V)\right)$.

(v) $\Rightarrow(\mathbf{i})$ :Let $V$ be any semi* $\alpha$-open set in $Y$. Then by assumption, $\operatorname{Int} *\left(\alpha C l\left(f^{1}(V)\right)\right)=\operatorname{Int} *\left(f^{-1}(V)\right)$. By Theorem 2.12, $f^{-1}(V)$ is semi* $\alpha$-closed in $X$. 
Theorem 4.12: (i) Every strongly semi* $\alpha$-irresolute function is semi* $\alpha$-irresolute and hence semi* $\alpha$-continuous.

(ii) Every semi $\alpha^{* *}$-continuous function is strongly semi* $\alpha$ irresolute.

Proof: $\quad$ Let $f: X \rightarrow Y$ be strongly semi* $\alpha$-irresolute. Let $V$ be semi* $\alpha$-open in $Y$. Since $f$ is strongly semi* $\alpha$-irresolute, $f^{1}(V)$ is open in $X$. Then by Theorem 2.8(ii), $f^{1}(V)$ is semi* $\alpha$-open. Therefore $f$ is semi* $\alpha$-irresolute. Hence by Theorem 4.6, $f$ is semi* $\alpha$-continuous.

Theorem 4.13: Every constant function is strongly semi* $\alpha$ irresolute.

Proof: Let $f: X \rightarrow Y$ be a constant function defined by $f(x)=y_{0}$ for all $x$ in $X$, where $y_{0}$ is a fixed point in $Y$. Let $V$ be a semi* $\alpha$-open set in $Y$. Then $f^{-1}(V)=X$ or $\phi$ according as $y_{0} \in V$ or $y_{0} \notin V$. Thus $f^{-1}(V)$ is open in $X$. Hence $f$ is strongly semi* $\alpha$-irresolute.

Theorem 4.14: Let $f: X \rightarrow Y$ be a function. Then the following are equivalent: (i) $f$ is strongly semi* $\alpha$-irresolute.

(ii) $f^{-1}(F)$ is closed in $X$ for every semi* $\alpha$-closed set $F$ in $Y$.

(iii) $f(C l(A)) \subseteq s^{*} \alpha C l(f(A))$ for every subset $A$ of $X$.

(iv) $C l\left(f^{-1}(B)\right) \subseteq f^{-1}\left(s^{*} \alpha C l(B)\right)$ for every subset $B$ of $Y$.

(v) $f^{-1}\left(s^{*} \alpha \operatorname{Int}(B)\right) \subseteq \operatorname{Int}\left(f^{-1}(B)\right)$ for every subset $B$ of $Y$.

Proof: (i) $\Rightarrow$ (ii): Let $F$ be a semi* $\alpha$-closed set in $Y$. Then $V=h Y$ is semi* $\alpha$-open in $Y$. Then $\quad f^{-1}(V)$ is open in $\mathrm{X}$. Therefore $f^{-1}(F)=f^{-1}(Y V)=X \backslash f^{-1}(V)$ is closed.

(ii) $\Rightarrow$ (i): $\quad$ Let $\mathrm{V}$ be a semi* ${ }^{*} \alpha$-open set in $\mathrm{Y}$. Then $\mathrm{F}=\mathrm{Y} \backslash \mathrm{V}$ is semi* $\alpha$-closed. By (ii), $f^{-1}(F)$ is closed. Hence $f^{-1}(V)=$ $f^{-1}(Y F)=X \backslash f^{-1}(F)$ is open in $X$. Therefore $f$ is strongly semi* $\alpha$ irresolute.

(ii) $\Rightarrow$ (iii): Let $A \subseteq X$. Let $F$ be a semi* $\alpha$-closed set containing $f(A)$. Then by (ii),

$f^{-1}(F)$ is a closed set containing $A$. This implies that $C l(A) \subseteq f$ ${ }^{1}(F)$ and hence $f(C l(A)) \subseteq F$. Therefore $f(C l(A)) \subseteq \mathrm{S} * \alpha C l(f(A))$.

(iii) $\Rightarrow\left(\right.$ iv): Let $B \subseteq Y$ and let $A=f^{-1}(B)$. By assumption, $(C l(A)) \subseteq \mathrm{S}^{*} \alpha C l(f(A)) \subseteq \mathrm{S}^{*} \alpha C l(B)$. This implies that $C l(A) \subseteq$ $f^{-1}\left(\mathrm{~s}^{*} \alpha C l(B)\right)$.

(iv) $\Rightarrow$ (ii): Let $\mathrm{F}$ be semi* $\alpha$-closed in Y. Then by Theorem 2.13(i), $\mathrm{s}^{*} \alpha C l(F)=F$. Therefore (iv) implies $C l\left(f^{-1}(F)\right) \subseteq$

$f^{-1}(F)$. Hence $C l\left(f^{-1}(F)\right)=f^{-1}(F)$. Therefore $f^{-1}(F)$ is closed.

(iv) $\Leftrightarrow(\mathbf{v})$ : The equivalence of (iv) and (v) follows from taking the complements.

Theorem 4.15: For a function $f: X \rightarrow Y$, the following are equivalent:

(i) $f$ is contra-strongly semi* $\alpha$-irresolute.

(ii)The inverse image of each semi* $\alpha$-closed set in $Y$ is open in $X$.

(iii)For each $x \in X$ and each semi* $\alpha$-closed set $F$ in $Y$ with $f(x) \in F$, there exists a open set $U$ in $X$ such that $x \in U$ and $f(U) \subseteq F$.

Proof:(i) $\Rightarrow$ (ii): Let $F$ be a semi* $\alpha$-closed set in $Y$. Then $Y F$ is semi* $\alpha$-open in $Y$. Since $f$ is contra-strongly semi* $\alpha$-irresolute, $f^{-1}(\backslash F)=X \backslash f^{-1}(F)$ is closed in $X$. Hence $f^{-1}(F)$ is open in $X$. This proves (ii).

(ii) $\Rightarrow$ (i): Let $U$ be a semi* $\alpha$-open set in $Y$. Then $Y U$ is semi* $\alpha$-closed in $Y$. By assumption, $\quad f^{-1}(Y U)=X \backslash f^{-1}(U)$ is closed in $X$. Hence $f^{-1}(U)$ is open in $X$.

(ii) $\Rightarrow$ (iii): Let $F$ be a semi* $\alpha$-closed set in $Y$ containing $f(x)$. Then $U=f^{-1}(F)$ is an open set containing $x$ such that $f(U) \subseteq F$. (iii) $\Rightarrow$ (ii): Let $F$ be a semi* ${ }^{*} \alpha$-closed set in $Y$ and $x \in f^{-1}(F)$, then $f(x) \in F$. By assumption, there is an open set $U_{x}$ in $X$ containing $x$ such that $f(x) \in f\left(U_{x}\right) \subseteq F$ which implies that $x \in U_{x} \subseteq f$ ${ }^{1}(F)$. Hence $f^{-1}(F)$ is open in $X$.

Theorem 4.16: (i) Composition of semi* $\alpha$-irresolute functions is semi* $\alpha$-irresolute.

(ii) Inverse of a bijective semi* $\alpha$-irresolute function is also semi* $\alpha$-irresolute.

Proof: Follows from definition and set theoretic results.

\section{MORE FUNCTIONS ASSOCIATED WITH SEMI* $\alpha$-OPEN SETS}

Definition 5.1: A function $\mathrm{f}: \mathrm{X} \rightarrow \mathrm{Y}$ is said to be semi* $\alpha$-open if $f(U)$ is semi* $\alpha$-open in $Y$ for every open set $U$ in $X$.

Definition 5.2: A function $f: X \rightarrow Y$ is said to be contrasemi* $\alpha$-open if $f(U)$ is semi* $\alpha$-closed in $Y$ for every open set $U$ in $X$.

Definition 5.3: A function $f: X \rightarrow Y$ is said to be pre-semi* $\alpha$ open if $f(U)$ is semi* $\alpha$-open in $Y$ for every semi* $\alpha$-open set $U$ in $X$.

Definition 5.4: A function $f: X \rightarrow Y$ is said to be contra-presemi* $^{*} \alpha$-open if $f(U)$ is semi* $\alpha$-closed in $Y$ for every semi* $\alpha$ open set $U$ in $X$.

Definition 5.5: A function $f: X \rightarrow Y$ is said to be semi* $\alpha$ closed if $f(F)$ is semi* $\alpha$-closed in $Y$ for every closed set $F$ in $X$.

Definition 5.6: A function $f: X \rightarrow Y$ is said to be contrasemi* $\alpha$-closed if $f(F)$ is semi* $\alpha$-open in $Y$ for every closed set $F$ in $X$.

Definition 5.7: A function $f: X \rightarrow Y$ is said to be pre-semi* $\alpha$ closed if $f(F)$ is semi* $\alpha$-closed in $Y$ for every semi* $\alpha$-closed set $F$ in $X$.

Definition 5.8: A function $f: X \rightarrow Y$ is said to be contra-presemi* $\alpha$-closed if $f(F)$ is semi* $\alpha$-open in $Y$ for every semi* $\alpha$ closed set $F$ in $X$.

Definition 5.9: A bijection $f: X \rightarrow Y$ is called a semi* $\alpha$ homeomorphism if $f$ is both semi* $\alpha$-irresolute and presemi* $\alpha$-open. The set of all semi* $\alpha$-homeomorphisms of $(X$, $\tau)$ into itself is denoted by $\mathrm{s}^{*} \alpha \mathrm{H}(X, \tau)$.

Definition 5.10: A function $f: X \rightarrow Y$ is said to be semi* $\alpha$ totally continuous if $f^{-1}(V)$ is clopen in $X$ for every semi* $\alpha$ open set $V$ in $Y$.

Definition 5.11: A function $f: X \rightarrow Y$ is said to be totally semi* $\alpha$-continuous if $f^{-1}(V)$ is semi* $\alpha$-regular in $X$ for every open set $V$ in $Y$.

Theorem 5.12: (i) Every pre-semi* $\alpha$-open function is semi* $\alpha$-open

(ii) Every semi* $\alpha$-open function is semi $\alpha$-open.

(iii) Every contra-pre-semi* $\alpha$-open function is contra-semi* $\alpha$ open.

(iv) Every pre-semi* $\alpha$-closed function is semi* $\alpha$-closed.

(v) Every contra-pre-semi* $\alpha$-closed function is contrasemi* $\alpha$-closed. 
Proof: Follows from definitions, Theorem 2.8 and Remark 2.9.

Theorem 5.13: Let $f: X \rightarrow Y$ and be $g: Y \rightarrow Z$ be functions. Then (i) $g \circ f$ is pre-semi* $\alpha$-open if both $\mathrm{f}$ and $\mathrm{g}$ are presemi* $\alpha$-open.

(ii) $g \circ f$ is semi* $\alpha$-open if $\mathrm{f}$ is $\operatorname{semi}^{*} \alpha$-open and $\mathrm{g}$ is presemi* $\alpha$-open.

(iii) $g \circ f$ is pre-semi* $\alpha$-closed if both $\mathrm{f}$ and $\mathrm{g}$ are pre-semi* $\alpha$ closed.

(iv) $g \circ f$ is semi* $\alpha$-closed if both $\mathrm{f}$ is semi* $\alpha$-closed and $\mathrm{g}$ is pre-semi* $\alpha$-closed.

Proof: Follows from definitions.

Theorem 5.14: Let $f: X \rightarrow Y$ be a function where $X$ is an Alexandroff space and $Y$ is any topological space. Then the following are equivalent:

(i) $f$ is semi* $\alpha$-totally continuous.

(ii) For each $x \in X$ and each semi* $\alpha$-open

set $V$ in $Y$ with $f(x) \in V$, there exists a clopen

set $U$ in $X$ such that $x \in U$ and $f(U) \subseteq V$.

Proof: (i) $\Rightarrow$ (ii): Suppose $f: X \rightarrow Y$ is semi* $\alpha$-totally continuous. Let $x \in X$ and let $V$ be a semi* $\alpha$-open set containing $f(x)$. Then $U=$

$f^{-1}(V)$ is a clopen set in $X$ containing $x$ and hence $f(U) \subseteq V$.

(ii) $\Rightarrow(\mathbf{i})$ : Let $V$ be a semi* $\alpha$-open set in $Y$. Let $x \in f^{-1}(V)$. Then $V$ is a semi* $\alpha$-open set containing $f(x)$. By hypothesis there exist a clopen set $U_{x}$ containing $x$ such that $f\left(U_{x}\right) \subseteq V$ which implies that $U_{x} \subseteq f^{-1}(V)$. Therefore we have

$f^{-1}(V)=\cup\left\{U_{x}: x \in f^{-1}(V)\right\}$. Since each $U_{x}$ is open, $f^{-1}(V)$ is open. Since each $U_{x}$ is a closed set in the Alexandroff space $X$, $f^{-1}(V)$ is closed in $X$. Hence $f^{-1}(V)$ is clopen in $X$.

Theorem 5.15: A function $f: X \rightarrow Y$ is semi* $\alpha$-totally continuous if and only if

$f^{1}(F)$ is clopen in $X$ for every semi* $\alpha$-closed set $F$ in $Y$.

Proof: Follows from definition.

Theorem 5.16: A function $f: X \rightarrow Y$ is totally semi* $\alpha$ continuous if and only if $f$ is both semi* $\alpha$-continuous and contra-semi* $\alpha$-continuous.

Proof: Follows from definitions.

Theorem 5.17: A function $f: X \rightarrow Y$ is semi* $\alpha$-totally continuous if and only if $f$ is both strongly semi* $\alpha$-irresolute and contra-strongly semi* $\alpha$-irresolute.

Proof: Follows from definitions.

Theorem 5.18: Let $f: X \rightarrow Y$ be semi* $\alpha$-totally continuous and $A$ is a subset of $Y$. Then the restriction $f_{A}: A \nmid Y$ is semi* $\alpha$ totally continuous.

Proof: Let $V$ be a semi* ${ }^{*} \alpha$-open set in $Y$. Then $f^{-1}(V)$ is clopen in $X$ and hence

$\left(\left.f\right|_{A}\right)^{-1}(V)=A \cap f^{-1}(V)$ is clopen in $A$. Hence the theorem follows.

Theorem 5.19: Let $f: X \rightarrow Y$ be a bijection. Then the following are equivalent: (i) $f$ is semi* $\alpha$-irresolute.

(ii) $f^{-1}$ is pre-semi* $\alpha$-open.

(iii) $f^{-1}$ is pre-semi* $\alpha$-closed.

Proof: Follows from definitions.

Theorem 5.20: A bijection $f: X \rightarrow Y$ is a semi* $\alpha$ homeomorphism if and only if $f$ and $f^{-1}$ are semi* $\alpha$ irresolute.

Proof: Follows from definitions.

Theorem 5.21: (i) The composition of two semi* $\alpha$ homeomorphisms is a semi* $\alpha$-homeomorphism

(ii) The inverse of a semi* $\alpha$-homeomorphism is also a semi* $\alpha$-homeomorphism.
Proof: (i) Let $f: X \rightarrow Y$ and $g: Y \rightarrow Z$ be semi* $\alpha$ homeomorphisms. By Theorem 4.16 and theorem 5.13(i), $g \circ f$ is a semi* $\alpha$-homeomorphism.

(ii)Let $f: X \rightarrow Y$ be a semi* $\alpha$-homeomorphism. Then by Theorem 4.16(ii) and by Theorem $5.20, f^{-1}: Y \rightarrow X$ is also semi* $\alpha$ homeomorphism.

Theorem 5.22: If $(X, \tau)$ is a topological space, then the set $\mathrm{s}^{*} \alpha \mathrm{H}(X, \tau)$ of all semi* $\alpha$-homeomorphisms of $(X, \tau)$ into itself forms a group.

Proof: Since the identity mapping $I$ on $X$ is a semi* $\alpha$ homeomorphism, $\mathrm{I} \in \mathrm{s} * \alpha \mathrm{H}(X, \tau)$ and hence $\mathrm{s}^{*} \alpha \mathrm{H}(X, \tau)$ is nonempty and the theorem follows from Theorem 5.21.

\section{REFERENCES}

[1] Levine, N., Semi-open sets and Semi-continuity in topological spaces, Amer. Math.Monthly, 70(1) (1963), 3641.

[2] Dontchev, J., Contra-continuous functions and strongly s-closed spaces, Internat. J. Math. \& Math. Sci. 19(2) (1996), 303-310.

[3] Crossley, S.G and Hildebrand, S.K., Semi-topological properties, Fund. Math.74 (1972), 233-254.

[4] Dontchev, J., and Noiri, T.,Contra-Semi continuous functions, Mathematica Pannonica, 10(2) (1999), 159-168.

[5] Caldas M., Weak and Strong forms of irresolute maps, Internat. J. Math. \& Math. Sci. 23(4) (2000) 253-259.

[6] Pasunkili Pandian,S., A Study on Semi Star-Preopen Sets in Topological Spaces, Ph.D Thesis, Manonmaniam Sundaranar University, Tirunelveli, India, 2013.

[7] Robert, A. and Pious Missier, S., A New Class of sets weaker than $\alpha$-open sets, International Journal of Mathematics and Soft Computing, 4(2) (2014), 197-206.

[8] Robert, A and Pious Missier, S., Between $\alpha$-closed sets and semi $\alpha$-closed sets, International Journal of Modern Engineering Research, 4(6) 2014, 34-41.

[9] Pious Missier, S and Robert, A., New Notions via SemiStar-Alpha-Open Sets. (communicated)

[10] Levine, N., Generalized Closed Sets in Topology, Rend. Circ. Mat. Palermo, 19(2) (1970), 89-96.

[11] Dunham, W., A new closure operator for Non-T topologies, Kyungpook Math. J. 22 (1) (1982), 55-60.

[12] Njastad,O., Some Classes of Nearly Open sets, $\quad P a-$ cific J. Math.,15 (3) (1965),961-970.

[13] Navalagi, G.B., Definition Bank in General Topology, Topology Atlas (2000).

[14] Andrijevic,D., Semi-Preopen Sets, Mat. Vesnik, 38(1986), 24-32.

[15] Othman, H. A., New types of $\alpha$-continuous mapping, Master Degree Thesis, Al-Mustansiriya University, IRAQ (2004)

[16] Nour.T.M., Totally semi-continuous functions, Indian J. Pure Appl. Math., 26(7) (1995), 675-678.

[17] Benchalli. S.S.and Umadevi I Neeli., Semi-Totally Continous Functions in Topological spaces, International Mathematical Forum, 6 (10) (2011), 479-492. 